\title{
Processing demand modulates the effects of spatial attention on the judged duration of a brief stimulus
}

\author{
ZHE CHEN and PATRICIA O'NEILL \\ University of Mississippi, University, Mississippi
}

\begin{abstract}
How does attention influence the judged duration of a brief stimulus? In the four experiments reported here, we show that the effect of spatial attention on duration judgment depends on the processing demand of the concurrent nontemporal task. When participants had to perform a speeded letter discrimination task in addition to duration rating, the judged duration was longer at a cued location than at an uncued location, regardless of whether the cue was exogenous or endogenous. However, when the same stimuli were presented but no concurrent nontemporal task was required, duration was judged to be shorter at the cued location, as compared with the uncued locations. Furthermore, although spatial attention influenced duration judgment, no object-based attentional effects were found. These findings suggest that, although spatial attention plays an important role in the judged duration of a briefly presented stimulus, its effect is mediated by the processing demand of the task.
\end{abstract}

One of the central questions in vision research is how attention influences visual information processing. $\mathrm{Nu}-$ merous studies have shown that the directing of attention to a spatial location or to an object increases the speed and/or accuracy of processing stimuli there relative to elsewhere (e.g., Baylis \& Driver, 1993; Chen, 1998b, 2000b; Duncan, 1984; Egly, Driver, \& Rafal, 1994; Hoffman \& Nelson, 1981; Posner, Snyder, \& Davidson, 1980; Shaw \& Shaw, 1977). Furthermore, attention reduces response variability (e.g., Prinzmetal, Amiri, Allen, \& Edwards, 1998; Prinzmetal \& Wilson, 1997) and enhances signal-to-noise ratio of a briefly presented stimulus (e.g., Bashinski \& Bacharach, 1980; C. J. Downing, 1988). These effects suggest that attention acts to enhance visual information processing.

However, recent studies suggest that the effect of attention on visual information processing may be more varied than had been previously appreciated. For example, Yeshurun and Carrasco (1998) showed that, whereas attention facilitates texture segregation at peripheral locations, it impairs it at central locations. Chen (2000c) reports that although spatial attention reduces response interference from incompatible distractors when target selection does not require a narrow attentional window,

We thank Ronald Kinchla, Anne Treisman, and Wei Yang for useful discussions of the data, and thank Steven Yantis, Stefan Mattes, Dominique Lamy, and an anonymous reviewer for helpful comments on an earlier version of the manuscript. We also thank the members of the Attention and Consciousness seminar at the University of Mississippi, Fall 1999, for suggestions that improved the manuscript. Correspondence concerning this article should be addressed to Z. Chen, Department of Psychology, University of Canterbury, Private Bag, Christchurch, New Zealand (e-mail: z.chen@psyc.canterbury.ac.nz). the effect is abolished when the task is made more difficult so that a narrow attentional window is needed to complete the task. Furthermore, allocating attention to an incompatible color word (e.g., the word RED written in green ink and the task is to identify the color of the ink as quickly as possible) increases the Stroop interference effect (Stroop, 1935) rather than reducing it (Chen, 2000a). These findings indicate that how attention influences visual information processing may depend on such factors as the nature of the task and the spread of attention during task completion.

In addition to the factors mentioned above, the efficiency of selective attention appears to be modulated by the perceptual load involved in the processing of the target. Lavie and her colleagues (e.g., Lavie, 1995, 2000; Lavie \& Cox, 1997; Lavie \& Tsal, 1994) have proposed that high perceptual load for a task is a necessary requirement for efficient selective attention and that perception proceeds automatically to the extent of available resources. When the processing of relevant task information does not consume all available resources (described as a lowload condition), the processing of task-irrelevant information will continue until all resources are used, and this will lead to high distractor interference. In contrast, when the processing load of the relevant task is high (the highload condition), no spare resources are available to process the task-irrelevant information. Hence, little distractor interference occurs. For example, in one experiment, Lavie and Cox asked participants to search for a target letter displayed simultaneously with a compatible, a neutral, or an incompatible irrelevant letter in the periphery. Participants suffered more interference from the incompatible letter than from the neutral letter when the rest of the items in the display consisted of homogeneous distractors such 
as Os (the low-load condition) rather than heterogeneous distractors such as $\mathrm{H}, \mathrm{W}, \mathrm{M}, \mathrm{Z}$, or $\mathrm{K}$, and so on (the highload condition). Therefore, attention may facilitate task performance or interfere with task performance, and the efficiency of selective attention is moderated by the processing demands of the task.

To date, most students of attention focus on how attention influences the appearance of, or the responses to, visual stimuli. Only a few researchers have directly examined the effects of attention on the processing of temporal characteristics of a briefly presented stimulus, and the main finding has been that spatial attention directed to a stimulus prolongs the judged duration of that stimulus (e.g., Enns, Brehaut, \& Shore, 1999; Mattes \& Ulrich, 1998). In a recent study, Mattes and Ulrich used precues to manipulate attention explicitly. They showed participants a briefly presented black dot for one of two intervals after an informative endogenous precue, and the cue validity was varied across blocks. The task was to categorize the stimulus duration as short, medium, or long. Among other important findings, the result most relevant to the present study is that observers' judged duration increased with stimulus probability. Similar results were also obtained in a subsequent dual-task experiment, in which observers compared the stimulus duration of a target dot at either a cued or an uncued location with the stimulus duration of a previously presented comparison dot at fixation. Before the duration judgment task, observers had to perform a speeded dot detection task. Again, the stimulus duration was judged to be longer at the cued location, as compared with the uncued location. At first glance, these results appear to suggest that attention increases the judged duration of a brief stimulus regardless of the nature and the processing demand of a task. However, processing demand was not the primary focus of the study and many methodological differences exist between these two experiments. Given this, it was difficult to determine whether processing demand could modulate the effect of attention on duration judgment.

In the series of experiments reported here, we explored the role of processing demand in modulating the effect of attention on duration judgments. We did so by asking participants to perform either single or dual tasks while presenting them with identical stimulus displays. In Experiment 1, we employed a dual-task paradigm. Observers performed a letter discrimination task as a primary task and a duration rating task as a secondary task. We used an exogenous cue and manipulated observers' allocation of both space- and object-based attention. Although object-based attention has been found in a variety of studies using reaction time and/or accuracy as dependent measures (e.g., Baylis \& Driver, 1993; Chen, 1998b, 2000b; Duncan, 1984; Egly et al., 1994; Moore, Yantis, \& Vaughan, 1998), the question of whether object-based attention could also influence duration judgment has not been addressed before. In Experiment 2, we investigated whether the attentional effect found in Experiment 1 was modulated by the presence of a concurrent, high pro- cessing demand, nontemporal second task. Observers performed the same duration rating task without letter discrimination. Experiments 3 and 4 generalized the results of the first two experiments with endogenous cues in order to confirm that the results were not due to other factors such as misattribution or forwarding masking.

\section{EXPERIMENT 1}

In Experiment 1, we investigated the role of attention in the duration judgment of a brief stimulus by using an exogenous cue in a dual-task paradigm. Stimulus displays were made up of two rectangles, either vertically or horizontally aligned, as is shown in Figure 1. After a precue at one of the four end locations of the rectangles, an

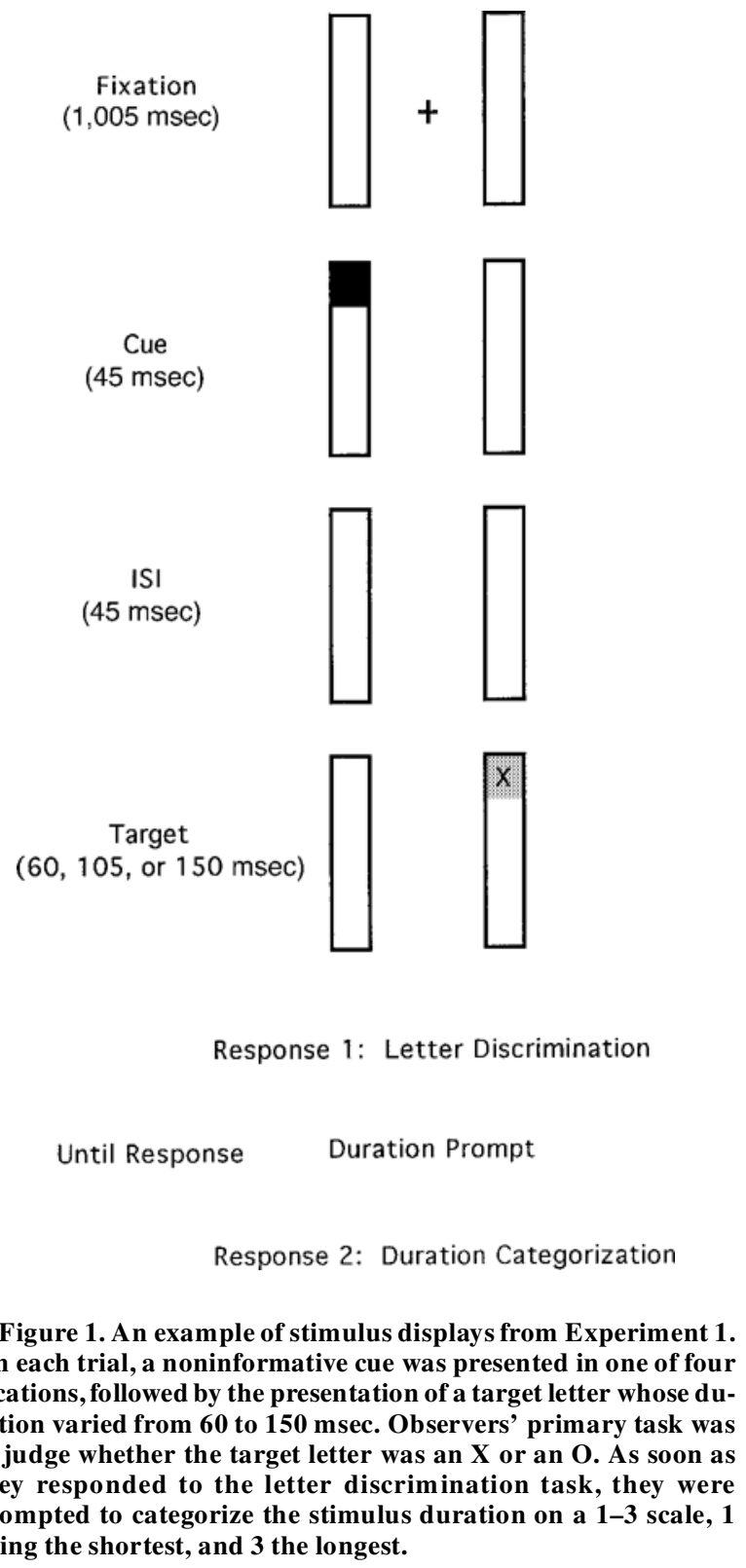


$\mathrm{X}$ or an $\mathrm{O}$ appeared either at the cued location or at an uncued location for one of three durations. The participants performed a speeded letter discrimination task, followed by duration rating of the target stimulus. Of particular interest was the participants' judged duration as a function of the cue-target relationship.

\section{Method}

Participants. Thirty undergraduate students from the University of Mississippi participated in the study to satisfy course requirements of the psychology department. All reported to have normal color vision.

Apparatus and Stimuli. A Power Macintosh 6100/66 computer with a 13-in. RGB monitor was used to present stimuli and to record responses. The participants viewed the monitor from a distance of approximately $60 \mathrm{~cm}$ in a dim room. A commercially available graphic program (Superpaint 3.0) and experimental program (MacProbe 1.6.9) were used to generate and display stimuli and to record responses.

The stimulus display consisted of two white outlined rectangles against a homogeneous black background. The rectangles were either horizontally or vertically aligned. At a viewing distance of approximately $60 \mathrm{~cm}$, each rectangle subtended $10.2^{\circ}$ of visual angle in length and $1.8^{\circ}$ in width. With a separation of $6.6^{\circ}$ between the inner contours of the rectangles, the entire stimulus display subtended $10.2^{\circ}$ in both length and width. The stimulus display was presented either alone or with one of three other stimuli: a fixation cross, a cue, or a letter. Both the fixation cross and the cue were white, and the letter was green. The fixation was placed at the center of the display, and it subtended $0.95^{\circ}$ of visual angle. The two letters used in the experiment were uppercase $\mathrm{X}$ and $\mathrm{O}$ (font, Geneva; point size, 48), and each had a radius of about $0.7^{\circ}$. Each trial contained only one letter, which was always presented against a small red square subtending $1.6^{\circ}$ of visual angle. The cue subtended $1.8^{\circ}$ of visual angle in both length and width.

Design and Procedure. The experiment used a repeated measures design. The variables of interest were stimulus duration (short, medium, and long) and the location of the target relative to the cue (same object-same location, same object-different location, different object-near location, and different object-far location). Altogether there were 12 experimental conditions, with each duration having four cue-target locations: same object-same location (SS), when the target appeared in the cued location; same object-different location (SD), when the cue and the target were in the same rectangle but different locations; different object-near location (DN), when the cue and the target were in different objects and the target was in the near end of the other rectangle from the cue; and different object-far location (DF), when the cue and the target were in different objects and the target was in the far end of the other rectangle from the cue.

Each trial started with the presentation of the fixation cross together with either two horizontal or vertical rectangles for $1,005 \mathrm{msec}$. The stimulus display was presented at the center of the screen, with the fixation cross at the center of the display. Upon the offset of the fixation (the rectangles remained on the screen throughout the trial), the cue was flashed for $45 \mathrm{msec}$ at one of the four ends of the rectangles. After the termination of the cue, the rectangles remained on the screen for another $45 \mathrm{msec}$ before a target letter appeared. The letter could either be an X or an $\mathrm{O}$, and it appeared at one of the four end regions of the rectangles randomly with equal probability. Please note that the spatial separation between the cue and the target was identical in SD and DN conditions, both subtending $8.30^{\circ}$ of visual angle. The duration of the letter varied from trial to trial: 60,105 , or $150 \mathrm{msec}$. Upon the offset of the letter, the screen returned to its background black color until the participant responded. The participant was told to identify the target letter as quickly as possible. They used their right index and middle fingers to press one of the two labeled keys on the keyboard (the " $<$ " key for X, and the " >" key for O). Upon response, a sentence would appear on the screen asking the participants to rate the duration of the letter, $1=$ short, $2=$ medium, and $3=$ long. They were then required to use their left hands to do the duration task. Three fingers (i.e., the ring, middle, and index fingers) were used to press the $\mathrm{Z}, \mathrm{X}$, and $\mathrm{C}$ keys, which were labeled 1,2 , and 3 , respectively. The sentence remained on the screen until a response was given. No feedback was provided during the experiment, and the intertrial interval was $1.5 \mathrm{sec}$.

The participants were aware that the cue was not informative. The importance of maintaining fixation throughout the trial was emphasized. The letter discrimination task was characterized as a primary task, and both speed and accuracy were stressed. The duration categorization was described as a secondary task, and only accuracy was emphasized. Before the experiment started, the participants were shown examples of trials - four trials with short intervals first, followed by four trials with medium and long intervals. No letter discrimination was required at this stage. If the participants indicated that they could see differences in duration between these trials, they were allowed to proceed to the next practice session. Otherwise, the whole process was repeated. Most participants were able to accomplish this after two repetitions. The participants then completed a block of 30 trials in which both speeded letter discrimination and duration rating were required. The experimental session consisted of three blocks of 192 trials, with half of them containing an "X," and the other half an "O." Each letter occurred at one of the four end regions of the rectangles equally often, and there were as many long interval trials as there were medium interval and short interval ones. This resulted in 48 trials per condition for each participant. The total experiment took about 45-50 min to complete, and the participants were encouraged to take short breaks between the blocks.

\section{Results and Discussion}

Letter discrimination task. The reaction time (RT) and accuracy data are shown in Table 1 . A repeated measures analysis of variance (ANOVA) on RT showed a significant main effect for cue $[F(3,87)=77.94, p<.001]$,

Table 1

Mean Reaction Times (in Milliseconds) and Error Rates (Percent Incorrect) for the Letter Discrimination Task of Experiment 1

\begin{tabular}{|c|c|c|c|c|c|c|c|c|}
\hline \multirow[b]{3}{*}{ Duration } & \multicolumn{8}{|c|}{ Conditions } \\
\hline & \multicolumn{2}{|c|}{ DF } & \multicolumn{2}{|c|}{$\mathrm{DN}$} & \multicolumn{2}{|c|}{ SD } & \multicolumn{2}{|c|}{ SS } \\
\hline & $M$ & $S D \mathrm{~s}$ & $M$ & $S D \mathrm{~s}$ & $M$ & $S D \mathrm{~s}$ & $M$ & $S D \mathrm{~s}$ \\
\hline \multicolumn{9}{|c|}{ Reaction Times } \\
\hline Long & 487 & 74 & 494 & 79 & 491 & 81 & 457 & 73 \\
\hline Medium & 493 & 74 & 493 & 76 & 484 & 78 & 457 & 77 \\
\hline Short & 497 & 78 & 494 & 69 & 487 & 75 & 455 & 74 \\
\hline Mean overall & 492 & 74 & 494 & 73 & 487 & 76 & 457 & 73 \\
\hline \multicolumn{9}{|c|}{ Error Rates } \\
\hline Long & 9.4 & 8.3 & 7.2 & 7.2 & 7.6 & 6.9 & 6.8 & 7.3 \\
\hline Medium & 7.1 & 6.0 & 6.8 & 5.6 & 7.6 & 6.0 & 8.4 & 7.8 \\
\hline Short & 7.0 & 5.3 & 7.3 & 6.2 & 7.2 & 8.4 & 7.6 & 5.9 \\
\hline Mean overall & 7.8 & 5.9 & 7.1 & 5.5 & 7.5 & 6.5 & 7.6 & 6.3 \\
\hline
\end{tabular}

Note-DF, different object-far location; DN, different object-near location; SD, same object-different location; SS, same object-same location. Note that the standard deviations $(S D s)$ shown here represent the between-participants variability within a condition instead of the withinparticipants variability across conditions that is of interest in the present paper. 
with no main effect for duration $[F(2,58)<1]$, or duration $\times$ cue interaction $[F(6,174)=1.26, p>.2]$. Planned mean comparisons revealed faster RTs to targets at the cued location ( $\mathrm{SS}=457 \mathrm{msec}$ ) than at any of the uncued locations ( $\mathrm{SD}=487 \mathrm{msec}, \mathrm{DN}=494 \mathrm{msec}$, and $\mathrm{DF}=$ $492 \mathrm{msec})[t(29)=8.41, p<.001$, for comparison between SS and SD; $t(29)=12.47, p<.001$, for comparison between SS and DN; and $t(29)=16.68, p<.001$, for comparison between SS and DF]. In addition to these findings, the participants were also faster in the SD than the DN conditions $[t(29)=2.50, p<.02]$. The difference between the SD and DF conditions approached significance $[t(29)=1.98, p<.06]$. An ANOVA on the accuracy data found no main effects or interaction, suggesting no speed-accuracy trade-off between RT and accuracy.

Like previous studies in the field (e.g., Chen, 1998b; Egly et al., 1994; Moore et al., 1998), we found both a location effect and an object effect. The location effect was demonstrated by the faster RTs to targets in a cued location than in an uncued location, and the object effect was indicated by the faster RTs to targets in the uncued location of the same object, as compared with a different object. These RT data suggested that our manipulation of attention was effective.

Duration categorization task. The duration data are shown in Table 2. An ANOVA revealed significant main effects for both duration $[F(2,58)=62.78, p<.001]$ and cue $[F(3,87)=4.23, p<.01]$ but no significant interaction between the two $[F(6,174)=1.15, p>.3]$. Planned mean comparisons on duration showed that the participants could discriminate between different presentation durations and that they judged the duration of the stimulus to be longer when it was long (mean rating $=2.13$ ) relative to when it was medium [mean rating $=1.88, t(29)=$ $7.19, p<.001]$ or short [mean rating $=1.62 ; t(29)=8.18$, $p<.001]$. The difference between the medium and short duration conditions also reached significance $[t(29)=$ $7.73, p<.001]$. Because similar duration effects were found in all four experiments reported here, we will not provide detailed statistical information for the other experiments. More importantly, the judged duration of the stimulus was affected by the manipulation of attention. The participants rated the stimulus interval to be longer

Table 2

Mean Perceived Duration for the

Duration Categorization Task of Experiment 1

\begin{tabular}{|c|c|c|c|c|c|c|c|c|}
\hline \multirow[b]{3}{*}{ Duration } & \multicolumn{8}{|c|}{ Conditions } \\
\hline & \multicolumn{2}{|c|}{ DF } & \multicolumn{2}{|c|}{$\mathrm{DN}$} & \multicolumn{2}{|c|}{ SD } & \multicolumn{2}{|c|}{ SS } \\
\hline & $M$ & $S D \mathrm{~s}$ & $M$ & $S D \mathrm{~s}$ & $M$ & $S D \mathrm{~s}$ & $M$ & $S D \mathrm{~s}$ \\
\hline Long & 2.06 & .23 & 2.11 & .27 & 2.13 & .24 & 2.22 & .42 \\
\hline Medium & 1.81 & .25 & 1.84 & .22 & 1.85 & .23 & 2.01 & .38 \\
\hline Short & 1.57 & .30 & 1.56 & .28 & 1.60 & .28 & 1.75 & .35 \\
\hline Mean overall & 1.81 & .20 & 1.84 & .19 & 1.86 & .19 & 1.99 & .36 \\
\hline
\end{tabular}

Note-Ratings from 1 to 3 were used to categorize the stimulus duration, with $1=$ short, 2 = medium, and $3=$ long. DF, different object-far location; DN, different object-near location; SD, same object-different location; SS, same object-same location. in the cued location (mean rating $=1.99$ ) than in an uncued location (mean rating $=1.84$ ). Specifically, the stimulus in SS conditions was perceived to be longer than the same stimulus in DF conditions [mean rating $=1.81$; $t(29)=2.24, p<.02]$, or in DN conditions [mean rating $=1.84 ; t(29)=2.06, p<.05]$. The difference between SS and SD conditions (mean rating for SD $=1.86$ ) approached significance $[t(29)=1.79, p<.09]$. A significant difference was also found between SD and DF conditions $[t(29)=2.91, p<.001]$, although the difference between SD and DN conditions did not reach significance $[t(29)=1.56, p>.1]$.

Our duration data show that spatial attention increased the judged duration of a brief stimulus in the present paradigm. When participants had to perform a speeded letter discrimination task in addition to duration rating, the stimulus duration appeared to be longer at the cued location than at the uncued locations, despite the fact that the cue was uninformative.

Although a significant object effect was indicated in RT, no object effect was found in duration rating. The lack of an object effect in duration judgment was not totally unexpected. Many reaction time studies that include letter discrimination tasks similar to that employed in the present experiment have shown that an object effect is typically much smaller than a location effect (e.g., Chen, 1998b; Egly et al., 1994; Moore et al., 1998). Furthermore, an object effect seems to be sensitive to a number of factors that do not necessarily influence a location effect. For example, the object effect depends on the perceptual organization of a stimulus (Chen, 1998b), the size of the attended region (Lavie \& Driver, 1996; but see Lamy, 2000), the uniform connectedness of an object's surface (Kramer \& Watson, 1995) and the type of precue employed in the study (Arrington \& Dagenbach, 2000; Macquistan, 1997; Neely \& Dagenbach, 1996; but see Abrams \& Law, 2000). Given these characteristics, it is possible that duration rating might not be a sensitive enough measure for the manifestation of an object effect.

Our next question is whether the attentional effect on duration judgment would remain the same when participants do not have to perform the letter discrimination task. We tested this possibility by showing participants identical stimulus displays as those in Experiment 1, but asked them to perform only the duration rating task.

\section{EXPERIMENT 2}

\section{Methods}

Participants. Twenty-six new undergraduates from the same participant pool as before took part in the study. All had normal or corrected-to-normal vision and color vision. None knew the purpose of the experiment in advance.

Apparatus and Stimuli. Both the apparatus and stimuli were exactly the same as Experiment 1.

Design and Procedure. The design and procedure were identical to those of Experiment 1, with the exception that no letter discrimination was required of the participants. Upon the offset of the target display that contained either an $\mathrm{X}$ or an $\mathrm{O}$, the participants 
rated the duration of the letter regardless of which one they saw. Only accuracy was stressed.

As before, the experiment consisted of three blocks of 192 experimental trials, and it took approximately $40 \mathrm{~min}$ to finish.

\section{Results and Discussion}

The duration data are shown in Table 3. The results showed significant main effects of duration $[F(2,50)=$ $55.27, p<.001]$, cue $[F(2,50)=3.94, p<.02]$, and a duration $\times$ cue interaction $[F(6,150)=2.92, p<.02]$. Like in Experiment 1, the participants could discriminate the three types of duration (the mean ratings for the long, medium, and short duration were $2.15,1.91$, and 1.68 , respectively). However, in contrast to the previous experiment, they judged the stimulus duration to be shorter at the cued location relative to an uncued location (the mean ratings for the SS, SD, DN, and DF conditions were 1.75, $1.97,1.97$, and 1.98 , respectively). As for the duration $X$ cue interaction, a single factor repeated measures ANOVA reveal that for trials having long and medium duration intervals, a significant effect for cue was found $[F(3,75)=$ $6.02, p<.002$, and $F(3,75)=3.25, p<.03$, respectively]. For trials with long duration, planned mean comparison showed shorter duration judgment when the stimulus was at a cued location than when it was at an uncued location $[t(25)=2.42, p<.03$ for comparison between DF and SS; $t(25)=2.12, p<.05$ for comparison between DN and SS; and $t(25)=2.66, p<.02$, for comparison between $\mathrm{SD}$ and SS]. For trials with medium duration, the comparison between DF and SS, and DN and SS approached significance $[t(25)=1.98, p<.06$, and $t(25)=1.96, p<$ .07 , respectively]. An ANOVA did not reveal any reliable differences among conditions for trials with short duration $[F(3,75)=1.53, p>.2]$. Given the data, it appears that, in the present paradigm, attention affected duration judgment only when the presentation duration was relatively long.

The results of Experiment 2 suggest that, at least in the present paradigm, the presence or absence of a concurrent nontemporal task plays an important role in determining the direction of the attentional influence on the judged duration of a briefly presented stimulus. When the participants had to engage in a speeded letter discrim-

Table 3

Mean Perceived Duration for the Duration Categorization Task of Experiment 2

\begin{tabular}{|c|c|c|c|c|c|c|c|c|}
\hline \multirow[b]{3}{*}{ Duration } & \multicolumn{8}{|c|}{ Conditions } \\
\hline & \multicolumn{2}{|c|}{ DF } & \multicolumn{2}{|c|}{$\mathrm{DN}$} & \multicolumn{2}{|c|}{ SD } & \multicolumn{2}{|c|}{ SS } \\
\hline & $M$ & $S D \mathrm{~s}$ & $M$ & $S D \mathrm{~s}$ & $M$ & $S D \mathrm{~s}$ & $M$ & $S D \mathrm{~s}$ \\
\hline Lon & 2.22 & .30 & 2.23 & .29 & 2.24 & .22 & 1.92 & .55 \\
\hline Medium & 1.99 & .25 & 1.98 & .24 & 1.94 & .24 & 1.75 & .47 \\
\hline Short & 1.72 & .30 & 1.71 & .31 & 1.72 & .27 & 1.58 & .38 \\
\hline Mean overall & 1.98 & .23 & 1.97 & .22 & 1.97 & .20 & 1.75 & .44 \\
\hline
\end{tabular}

Note-DF, different object-far location; DN, different object-near location; SD, same object-different location; SS, same object-same location. ination task in addition to duration estimation, spatial attention prolonged the stimulus duration. In contrast, in the absence of a concurrent nontemporal task, spatial attention shortened judged duration.

Why is our result different from those of Mattes and Ulrich (1998), who reported increased stimulus duration at the cued location even when duration judgment was the sole task? The many methodological differences between Mattes and Ulrich's experiment and ours make it difficult to isolate the reason for the different results. One difference that readily comes to mind is the stimulus onset asynchrony (SOA) between the cue and the target. In our experiments, the cue-target SOA was $90 \mathrm{msec}$, whereas in Mattes and Ulrich, it was 2,000 msec. Although a longer SOA could allow participants to make eye movements, the fact that similar results were obtained when the authors monitored participants' eye movements makes it unlikely for SOA to be a contributing factor. Another difference between the two experiments is the specific stimuli used in the studies. Whereas we employed letters (X vs. O), Mattes and Ulrich chose dots. It is possible that, because the task load was low and letters are inherently more meaningful than dots, the participants in our experiments may have been processing some aspects of the stimuli even though letter discrimination was not required. This, however, does not mean that participants processed the letters in the same way as they did in Experiment 1. After all, whereas letter identification was the primary task in Experiment 1, no identification was needed in Experiment 2. Similar differences may underlie our experiments and those of Enns et al. (1999), who used dot stimuli in a different paradigm and found longer judged duration at the cued than at the uncued locations without a concurrent nontemporal task. Task load may have interacted with stimulus type in some way, leading to the observed differences among the various studies. At present, we are still unclear as to the nature of the interaction.

Even though the results of Experiments 1 and 2 demonstrate the importance of processing demand in the attentional effect on duration judgment, one might argue that there are confounds in our experiments. For example, the participants could have misattributed the exogenous cue as part of the target stimulus in the valid cue condition, leading to longer judged duration on valid than on invalid cue trials in Experiment 1 (see Witherspoon \& Allen, 1985, for a related phenomenon concerning judged duration and stimulus familiarity). These cues could also have induced forward masking effects that might have influenced duration judgment. Furthermore, because of the brief stimulus presentation duration and the short SOA between the cue and the target, the participants might experience apparent motion on invalid trials but not on valid trials, resulting in longer judged duration on invalid than on valid trials in Experiment 2. Even though none of these factors can adequately explain why we obtained the opposite pattern of results when identical stimulus 
displays were used in both experiments, nonetheless, it is important to see whether a similar pattern of results is obtained when endogenous cues are used. Endogenous cues should minimize these factors.

\section{EXPERIMENT 3}

\section{Methods}

Participants. Nineteen new participants were recruited from the same undergraduate participant pool at the University of Mississippi.

Apparatus and Stimuli. The apparatus remained the same as before. Several changes were made to the stimuli. First, to minimize the effect of visible persistence (Breitmeyer, 1984), all the stimuli, including the outline rectangles, the letters, and the precue were changed from white to black, and they were presented against a white background. Second, rather than an exogenous luminance cue, the new experiment employed an endogenous arrow cue. It subtended $2.96^{\circ}$ of visual angle and was located at the center of the display. Third, the small red square against which the target letter was displayed in Experiments 1 and 2 was not used in this experiment. All other aspects of the stimuli were identical to those employed in the first two experiments.

Design and Procedure. The only changes in regard to the design of the experiment concerned the cue validity and the number of experimental conditions. To encourage the participants to attend to the cue, valid cue trials comprised $60 \%$ of the total trials (the SS condition), with the remaining $40 \%$ of trials divided equally between the same object-different location condition (SD) and the different object-near location condition (DN). The different object-far location condition (DF) was not included in the experiment. With three levels of stimulus intervals (i.e., short, medium, and long), there were nine conditions altogether.

The experimental procedure was similar to Experiment 1. Each trial started with the presentation of a fixation cross, together with either two horizontal or vertical rectangles, and the rectangles remained throughout the trial. The fixation cross stayed on the screen for 1,005 msec, and 1,005 msec after its offset, the cue appeared for $45 \mathrm{msec}$. After an SOA of $90 \mathrm{msec}$, the target letter was displayed for one of three intervals: 60, 105, or $150 \mathrm{msec}$. As in Experiment 1, the participants made a two-alternative speeded response to the target letter before they rated its duration. The experiment was composed of four blocks of 120 trials, with 288 trials in the SS conditions and 96 trials each in the SD and DN conditions.

Table 4

Mean Reaction Times (in Milliseconds) and Error Rates (Percent Incorrect) for the Letter Discrimination Task of Experiment 3

\begin{tabular}{|c|c|c|c|c|c|c|}
\hline \multirow[b]{3}{*}{ Duration } & \multicolumn{6}{|c|}{ Conditions } \\
\hline & \multicolumn{2}{|c|}{$\mathrm{DN}$} & \multicolumn{2}{|c|}{ SD } & \multicolumn{2}{|c|}{ SS } \\
\hline & $M$ & $S D \mathrm{~s}$ & $M$ & $S D \mathrm{~s}$ & $M$ & $S D \mathrm{~s}$ \\
\hline \multicolumn{7}{|c|}{ Reaction Times } \\
\hline Long & 550 & 186 & 537 & 169 & 526 & 155 \\
\hline Medium & 529 & 157 & 535 & 165 & 517 & 157 \\
\hline Short & 545 & 157 & 536 & 152 & 515 & 153 \\
\hline Mean overall & 541 & 164 & 536 & 158 & 519 & 154 \\
\hline \multicolumn{7}{|c|}{ Error Rates } \\
\hline Long & 5.9 & 5.8 & 7.5 & 7.7 & 6.6 & 4.9 \\
\hline Medium & 9.2 & 9.7 & 8.3 & 6.9 & 8.0 & 6.4 \\
\hline Short & 8.3 & 8.1 & 7.8 & 7.4 & 7.5 & 6.3 \\
\hline Mean overall & 7.8 & 7.2 & 7.9 & 6.2 & 7.4 & 5.5 \\
\hline
\end{tabular}

Note-DN, different object-near location; SD, same object-different location; SS, same object-same location.
Table 5

Mean Perceived Duration for the Duration Categorization Task of Experiment 3

\begin{tabular}{|c|c|c|c|c|c|c|}
\hline \multirow[b]{3}{*}{ Duration } & \multicolumn{6}{|c|}{ Conditions } \\
\hline & \multicolumn{2}{|c|}{$\mathrm{DN}$} & \multicolumn{2}{|c|}{ SD } & \multicolumn{2}{|c|}{ SS } \\
\hline & $M$ & $S D \mathrm{~s}$ & $M$ & $S D \mathrm{~s}$ & $M$ & $S D \mathrm{~s}$ \\
\hline Long & 2.18 & .21 & 2.15 & .23 & 2.24 & .24 \\
\hline Medium & 1.89 & .20 & 1.88 & .24 & 1.95 & .17 \\
\hline Short & 1.51 & .27 & 1.61 & .33 & 1.62 & .32 \\
\hline Mean overall & 1.86 & .15 & 1.88 & .18 & 1.94 & .17 \\
\hline
\end{tabular}

Note-DN, different object-near location condition; SD, same objectdifferent location condition; SS, same object-same location condition.

\section{Results and Discussion}

The data for the letter discrimination task are shown in Table 4, and the duration ratings are in Table 5. Only 18 of the 19 participants' data were included in the analyses due to the excessively long RTs of 1 participant, whose mean response latencies were more than three standard deviations above the group mean.

Letter discrimination task. As expected, an ANOVA found a significant main effect of cue $[F(2,34)=4.81$, $p<.05]$, with no main effect of duration $[F(2,34)<1]$ or a duration $\times$ cue interaction $[F(4,68)<1]$. Like in Experiment 1 , the participants were faster at doing the letter discrimination task when the target letter occurred at the cued location $(R T=519 \mathrm{msec})$ than at an uncued location $(R T=536 \mathrm{msec}$ and $541 \mathrm{msec}$ for SD and DN conditions, respectively) $[t(17)=2.17, p<.05$, for comparison between SS and SD conditions, and $t(17)=3.20$, $p<.01$, for comparison between SS and DN conditions]. Paired comparison between the SD and DN conditions did not reach significance $[t(17)<1]$.

The lack of an object effect in the RT data in this experiment is consistent with prior reports in the literature. It is possible that different mechanisms are involved in different types of cues (Briand \& Klein, 1987; Jonides, 1981; Nakayama \& Mackeben, 1989). Therefore, although an object effect occurs with exogenous cues, it did not, in the present experiment, occur with endogenous cues, a result consistent with previous work (e.g., Arrington \& Dagenbach, 2000; Macquistan, 1997; Neely \& Dagenbach, 1996; but see Abrams \& Law, 2000).

Duration categorization task. There were significant main effects for duration $[F(2,34)=42.61, p<.001]$ and for cue $[F(2,34)=4.09, p<.05]$, with no duration $\times$ cue interaction $[F(4,68)<1]$. Like in Experiment 1 , the participants could readily discriminate the three types of duration (the mean ratings for the long, medium, and short durations were $2.19,1.91$, and 1.58 , respectively, and all pair-wise comparisons were significant). They also judged the stimulus interval to be longer at the cued location (1.94) than at either of the uncued locations $[1.88$ for the $\mathrm{SD}$ condition, $t(17)=2.11, p<.05$; and 1.86 for the DN condition, $t(17)=3.0, p<.01]$.

Changing from exogenous to endogenous cues reduced the likelihood that the attentional effects observed in Experiment 1 were contaminated by such factors as pre- 
cue misattribution or induced forward masking. Given that duration was judged to be longer at the cued location than at the uncued locations, regardless of cue type, we were reasonably confident that spatial attention increased the judged duration of a brief stimulus when the participants had to perform a speeded nontemporal task in addition to duration estimation.

\section{EXPERIMENT 4}

This experiment examined whether the reversed effect of attention on duration perception observed in Experiments 1 and 2 was due to the effect of apparent motion on invalid trials. The participants were shown displays identical to those in Experiment 3, and they performed the duration rating task without engaging in letter identification. Since the experiment contained centrally located arrow cues, the effect of apparent motion, if any, should exert similar influence on both the valid and invalid trials. Therefore, if the results of Experiment 2 were primarily due to apparent motion, we should not find any cuing effects. However, if the results of Experiment 2 could be attributed to our manipulation in processing demand, we should still be able to observe longer judged duration on invalid than on valid trials.

\section{Methods}

Participants. Twenty-five undergraduates from the same participant pool as before took part in the study to satisfy a course requirement. None had taken part in the other three experiments.

Apparatus and Stimuli. These were the same as those used in Experiment 3.

Design and Procedure. Other than the removal of the letter discrimination task, all other aspects of the experiment were identical to those of Experiment 3.

\section{Results and Discussion}

Table 6 contains the participants' data. One person did not complete the experiment, so the analyses were based on the remaining 24 people. An ANOVA indicated significant main effects for both duration $[F(2,46)=27.23$, $p<.001]$ and cue $[F(2,46)=3.49, p<.05]$, with no duration $\times$ cue interaction $[F(4,92)<1]$. As before, the participants could distinguish the three levels of stimulus duration (the mean ratings for the long, medium, and

Table 6

Mean Perceived Duration for the Duration Categorization Task of Experiment 4

\begin{tabular}{|c|c|c|c|c|c|c|}
\hline \multirow[b]{3}{*}{ Duration } & \multicolumn{6}{|c|}{ Conditions } \\
\hline & \multicolumn{2}{|c|}{$\mathrm{DN}$} & \multicolumn{2}{|c|}{$\mathrm{SD}$} & \multicolumn{2}{|c|}{ SS } \\
\hline & $M$ & $S D s$ & $M$ & $S D s$ & $M$ & $S D s$ \\
\hline$\overline{\text { Long }}$ & 2.21 & .23 & 2.16 & .24 & 2.12 & .17 \\
\hline Medium & 2.00 & .17 & 1.95 & .18 & 1.93 & .19 \\
\hline Short & 1.71 & .33 & 1.69 & .29 & 1.70 & .31 \\
\hline Mean overall & 1.97 & .14 & 1.93 & .14 & 1.92 & .13 \\
\hline
\end{tabular}

Note-DN, different object-near location condition; SD, same objectdifferent location condition; SS, same object-same location condition. short intervals were $2.16,1.96$, and 1.70 , respectively), and all pair-wise comparisons reached significance. More interestingly, as in Experiment 2, the participants rated the stimulus interval to be shorter at the cued location than at an uncued location (the mean rating for the SS, SD, and DN conditions were 1.92, 1.93, and 1.97, respectively). Paired comparison indicated a significant difference between the SS and DN conditions $[t(23)=2.29$, $p<.05]$. No other effects reached significance.

The results of this experiment replicated those of Experiment 2, although the attentional effect was much smaller and was only significant between the SS and DN conditions. It is worth noting that a reduction in the magnitude of the attention effect was also found from Experiment 1 to Experiment 3 when dual tasks were employed. Because an exogenous cue reaches the peak of its effect much more rapidly than an endogenous cue (Nakayama \& Mackeben, 1989), the cuing effect might not have been fully realized in the last two experiments, since SOAs were extremely short. However, despite reduced magnitude, the important thing is that the processing demand of a task has been found to mediate the effects of attention on duration judgment using both exogenous and endogenous cues.

\section{GENERAL DISCUSSION}

Prior research has shown that, in prospective duration estimation, when attention is manipulated through an endogenous precue (Enns et al., 1999; Mattes \& Ulrich, 1998), observers' judged duration is increased at the cued location, as compared with an uncued location. Using both exogenous and endogenous cues, we extended these previous findings in several ways. We provided converging evidence that a briefly presented stimulus at an attended region could be judged as longer than a stimulus at an unattended region, regardless of cuing type. More importantly, we found that the effects of attention depended on the processing demand of a task. In dual tasks, when processing demand was high, the increase in attention increased judged duration (Experiments 1 and 3). In contrast, in single tasks, when processing demand was low, increasing attention shortened judged duration (Experiments 2 and 4). Furthermore, duration judgments were not affected by object-based attention in our paradigms.

How can we account for the data? We believe that, as the level of processing demands can modulate the efficiency of selective attention (Lavie, 1995, 2000), it can also modulate the effect of attention on duration judgment in our experiments. Experiments 1 and 3 involved high processing load. According to Thomas and Weaver (1975), each stimulus is analyzed by two processors: a nontemporal information processor to encode the nontemporal features of a stimulus, and a timer to code the temporal information and to accumulate pulses that correlate positively with the passage of time. Due to limitations in our cognitive resources, attention has to be shared between these two processors. When more attention is devoted to 
one processor, less attention is available for the other. Insufficient attention could cause the loss of some pulses, leading to shorter judged duration. In our experiments, because the participants had to switch attention from the cued to the uncued location on invalid trials, some pulses could be lost, resulting in the shorter judged duration at an unattended location relative to an attended location.

Alternatively, attention could influence duration perception via its effect on the perceptual quality of the target stimulus. Since letter discrimination was the primary task, the participants might not have sufficient resources available to process the temporal cues of a stimulus duration (e.g., the onset/offset time of the target stimulus). Instead, they might infer the duration of a stimulus on the basis of its brightness or clarity. From prior research, we know that attention can make a brief stimulus appear brighter (e.g., Bashinski \& Bacharach, 1980; Downing, 1988), which in turn could make the stimulus interval appear longer (Goldstone, Lhamon, \& Sechzer, 1978) and the letter discrimination task easier. These effects were likely to result in longer judged duration at the cued location than at an uncued location. Although we cannot distinguish between these two interpretations in our experiments, we lean towards the second because of the data pattern of Experiments 2 and 4. It is unclear how Thomas and Weaver's (1975) model would explain the shorter judged duration at the cued location, as compared with the uncued location.

Assuming that the perceptual quality hypothesis is true, can we say that the effect we found in Experiments 1 and 3 is not an effect of attention but rather an effect of misattribution (i.e., participants mistakenly attributing the clearer or brighter stimulus as having a longer duration)? Although this question is legitimate, we believe it is more accurate to attribute the differential duration ratings to our attentional manipulation because we used physically identical stimulus displays on both valid and invalid trials. Whatever effect the perceptual quality of the letters had on the participants was not due to the manipulation of the perceptual quality, but rather to the manipulation of attention. In other words, differential perceptual quality of the letters is only a by-product of attention. It is the distribution of attention that influenced the perceptual quality of the stimulus letters, which in turn affected the perception of the stimulus duration.

In contrast to Experiments 1 and 3, the participants judged the stimulus duration to be shorter at the cued location than at the uncued location. How can we explain that? One possibility is that the reversed data pattern is caused by differences in task priority among the experiments. Whereas duration rating was a secondary task in Experiments 1 and 3, it was a primary task in Experiments 2 and 4 . There is evidence that duration judgment is influenced by task priority. Zakay (1998) showed that duration estimation was longer when it was a primary task than when it was a secondary task, and this priority effect was independent of the task complexity effect, which he also manipulated in the study. In our experi- ments, the overall rating of the stimulus interval was also numerically higher in Experiments 2 and 4 (1.92 and 1.94, respectively), when duration rating was a primary task, than in Experiments 1 and 3 (1.88 and 1.89, respectively), when it was a secondary task, even though the difference is not statistically significant $[F(1,54)=1$ for comparison between Experiments 1 and 2, and $F(1,40)=1.13$, $p<.20$ for comparison between Experiments 3 and 4]. Although we cannot determine from the present experiments whether the difference in task priority was a major contributor to our results, such an explanation does not seem likely. If task priority interacted with resource allocation, Zakay (1998) would have found a significant interaction between task priority and task complexity. Furthermore, in an experiment not reported here, we replaced the high demanding letter discrimination task of Experiment 1 with a less demanding letter detection task and still asked participants to do the duration rating as a secondary task. We found that the attentional effect on duration judgment varied with the processing demand.

Another possibility is to attribute the reversal of the data pattern from Experiments 1 and 3 to Experiments 2 and 4 to the differential level of processing load required in the experiments. Experiments 2 and 4 involved low processing load, since the participants were only asked to determine the presentation duration of the stimulus. Because letter identification was not needed, the participants might have been affected less by the clarity of the letter, but more by such temporal cues as the onset/offset time of the target stimulus. Spatial attention is known to speed up the detection of both stimulus onset (Chen, 1998a; Hikosaka, Miyauchi, \& Shimojo, 1993a, 1993b; Stelmach \& Herdman, 1991; Stelmach, Herdman, \& McNeil, 1994) and stimulus offset (Downing \& Treisman, 1997). In other words, to an observer, a stimulus could appear later as well as extinguish later on an invalid cued trial compared with a valid cued trial. If participants put more weight on their impressions of the offset time of a stimulus more than on its onset time when they performed the task, this would bias their duration judgments in the direction of invalid cued trials, leading to longer judged duration on those trials, as compared with valid cued trials. Currently, it is still unclear under what conditions participants would be influenced more by the onset of a stimulus rather than by the offset of a stimulus, or vice versa. Further experiments are needed to understand the interactions among attention, processing demand, and the judged duration of a briefly presented stimulus.

Our results are also related to the findings in the time perception literature, which show that temporal judgment is affected not only by the actual duration of an interval, but also by a variety of nontemporal factors (e.g., Hicks, Miller, \& Kinsbourne, 1976; Predebon, 1996a). In prospective timing where observers are aware of the duration judgment task before the judged interval starts, duration is judged to be longer when the to-be-judged interval is filled with complex stimuli than when it is not. 
For example, relative to an empty interval, observers report longer duration when the same interval is filled with words (e.g., Thomas \& Weaver, 1975), light (e.g., Goldfarb \& Goldstone, 1963), or tones (e.g., Buffardi, 1971; Ihle \& Wilsoncroft, 1983). Similarly, an interval containing more stimulus events tends to be experienced longer than the same interval containing fewer stimulus events (e.g., Buffardi, 1971; Mo, 1971, 1974, 1975), and the effect has been observed in visual, auditory, and tactile modalities (e.g., Buffardi, 1971). Intervals containing moving stimuli are also perceived to be longer than the same interval containing stationary stimuli, and the same holds true for intervals filled with flickering light rather than those filled with static light (e.g., Lhamon \& Goldstone, 1975).

In contrast, when participants have to engage in a concurrent nontemporal task in addition to duration estimation, the pattern of results reverses. The more complex the stimuli are, or the more difficult the nontemporal task is, the shorter the judged duration (e.g., Brown, 1985; Hicks, Miller, Gaes, \& Bierman, 1977; Hicks et al., 1976; Macar, 1996; McClain, 1983; Predebon, 1996a, 1996b; Zakay, 1993, 1998). For instance, when participants were asked to sort cards into one stack, two stacks (by color), or four stacks (by suit) during a 42 -sec interval, their judged duration decreased linearly from the one-stack condition to the four-stack condition even though task duration remained constant for all three conditions (Hicks et al., 1977; Hicks et al., 1976). Similarly, negative correlation has been reported between judged duration of an interval and the number of words to be classified (e.g., Predebon, 1996a, 1996b), the number of angles to be identified (e.g., Zakay, 1993), and the level of difficulty in a line-tracing task (e.g., Brown, 1985). Although it is difficult to directly compare our results with the findings from the timing literature due to many methodological differences, these data do suggest that the presence or absence of a secondary nontemporal task influences duration judgment.

To summarize, the present study supports the notion that attention plays an important role in duration judgment. However, how it affects the judged interval of a briefly presented stimulus depends on the processing demand in completing a task.

\section{REFERENCES}

Abrams, R. A., \& Law, M. B. (2000). Object-based visual attention with endogenous orienting. Perception \& Psychophysics, 62, 818-833.

Arrington, C. M., \& Dagenbach, D. (2000, November). The reliability of object-based attention following peripheral and central cues. Poster session presented at the 41st Annual Meeting of the Psychonomic Society, New Orleans.

BASHINSKI, H. S., \& BACHARACH, V. R. (1980). Enhancement of perceptual sensitivity as the result of selectively attending to spatial locations. Perception \& Psychophysics, 28, 241-248.

Baylis, G. C., \& Driver, J. (1993). Visual attention and objects: Evidence for hierarchical coding of location. Journal of Experimental Psychology: Human Perception \& Performance, 19, 451-470.

Breit meyer, B. G. (1984). Visual masking: An integrative approach. New York: Oxford University Press.

Briand, K. A., \& KLein, R. M. (1987). Is Posner's "beam" the same as
Treisman's "glue"? On the relation between visual orienting and feature integration theory. Journal of Experimental Psychology: Human Perception \& Performance, 13, 228-241.

Brown, S. W. (1985). Time perception and attention: The effects of prospective versus retrospective paradigms and task demands on perceived duration. Perception \& Psychophysics, 38, 115-124.

BUfFARDI, L. (1971). Factors affecting the filled-duration illusion in the auditory, tactual, and visual modalities. Perception \& Psychophysics, 10, 292-294.

CHen, Z. (1998a). Image structure, subjective organization and objectbased allocation of visual attention (Doctoral dissertation, Princeton University, 1998). Dissertation Abstracts International, 59, 1384.

Chen, Z (1998b). Switching attention within and between objects: The role of subjective organization. Canadian Journal of Experimental Psychology, 52, 7-16.

Chen, Z (2000a). The effect of attention and memory load on Stroop interference effect [ARVO Abstract]. Investigative Ophthalmology \& Visual Science, 41, S42. (Abstract number 216)

Chen, Z. (2000b). An object-based cost of visual filtering. Perception \& Psychophysics, 62, 482-495.

CHEN, Z (2000c, November). Spatial attention reduces response interference in low, but not in high, processing load tasks. Poster session presented at the 41st Annual Meeting of the Psychonomic Society, New Orleans.

DownIng, C. J. (1988). Expectancy and visual-spatial attention: Effects on perceptual quality. Journal of Experimental Psychology: Human Perception \& Performance, 14, 188-202.

Downing, P. E., \& Treisman, A. M. (1997). The line-motion illusion: Attention or impletion? Journal of Experimental Psychology: Human Perception \& Performance, 23, 768-779.

DunCAN, J. (1984). Selective attention and the organization of visual information. Journal of Experimental Psychology: General, 113, 501-517.

Egly, R., Driver, J., \& Rafal, R. D. (1994). Shifting visual attention between objects and locations: Evidence from normal and parietal lesion participants. Journal of Experimental Psychology: General, 123, 161-177.

Enns, J. T., Brehaut, M. C., \& Shore, D. I. (1999). The duration of a brief event in the mind's eye. Journal of General Psychology, 126, 355-372.

GoldFarb, J. L., \& Goldstone, S. (1963). The judgment: A comparison of filled and unfilled duration. Perceptual \& Motor Skills, 16, 376.

Goldstone, S., Lhamon, W. T., \& Sechzer, J. (1978). Light intensity and judged duration. Bulletin of the Psychonomic Society, 12, 83-84.

Hicks, R. E., Miller, G. W., Gaes, G., \& Bierman, K. (1977). Concurrent processing demands and the experience of time-in-passing. American Journal of Psychology, 90, 431-446.

Hicks, R. E., Miller, G. W., \& Kinsbourne, M. (1976). Prospective and retrospective judgments of time as a function of amount of information processed. American Journal of Psychology, 89, 719-730.

Hikosaka, O., Miyauchi, S., \& Shimojo, S. (1993a). Focal visual attention produces illusory temporal order and motion sensation. $\mathrm{Vi}$ sion Research, 33, 1219-1240.

Hikosaka, O., Miyauchi, S., \& Shimojo, S. (1993b). Voluntary and stimulus-induced attention detected as motion sensation. Perception, 22, 519-526.

Hoffman, J. E., \& Nelson, B. (1981). Spatial selectivity in visual search. Perception \& Psychophysics, 30, 283-290.

Ihle, R. C., \& Wilsoncroft, W. E. (1983). The filled-duration illusion: Limits of duration of interval and auditory fillers. Perceptual \& Motor Skills, 56, 655-660.

JoNIDES, L. (1981). Voluntary versus automatic control over the mind's eye's movement. In J. Long \& A. Baddeley (Eds.), Attention and performance IX (pp. 187-203). Hillsdale, NJ: Erlbaum.

Kramer, A. F., \& Watson, S. E. (1995). Object-based visual selection and the principle of uniform connectedness. In A. F. Kramer, M. G. H. Coles, \& G. D. Logan (Eds.), Converging operations in the study of visual selective attention (pp. 395-414). Washington, DC: American Psychological Association.

LAmY, D. (2000). Object-based selection under focused attention: A failure to replicate. Perception \& Psychophysics, 62, 1272-1279.

Lavie, N. (1995). Perceptual load as a necessary condition for selective 
attention. Journal of Experimental Psychology: Human Perception \& Performance, 21, 451-468.

LAVIE, N. (2000). Selective attention and cognitive control: Dissociating attentional function through different types of load. In S. Monsell \& J. Driver (Eds.), Attention and performance XVIII (pp. 175-194). Cambridge, MA: MIT press.

LAVIE, N., \& Cox, S. (1997). On the eff iciency of visual selective attention: Efficient visual search leads to inefficient distractor rejection. Psychological Science, 8, 395-398.

LAVIE, N., \& Driver, J. (1996). On the spatial extent of attention in objectbased visual selection. Perception \& Psychophysics, 58, 1238-1251.

Lavie, N., \& Tsal, Y. (1994). Perceptual load as a major determinant of the locus of selection in visual attention. Perception \& Psychophysics, 56, 183-197.

Lhamon, W. T., \& Goldstone, S. (1975). Movement and the judged duration of visual targets. Bulletin of the Psychonomic Society, 5, 53-54.

MACAR, F. (1996). Temporal judgments on intervals containing stimuli of varying quantity, complexity and periodicity. Acta Psychologica, 92, 297-308

Macquistan, A. D. (1997). Object-based allocation of visual attention in response to exogenous, but not endogenous, spatial precues. Psychonomic Bulletin \& Review, 4, 512-515.

Mattes, S., \& UlRICH, R. (1998). Directed attention prolongs the perceived duration of a brief stimulus. Perception \& Psychophysics, 60, 1305-1317.

MCCLAIN, L. (1983). Interval estimation: Effect of processing demands on prospective and retrospective reports. Perception \& Psychophysics, 34, 185-189.

Mo, S. S. (1971). Judgment of temporal duration as a function of numerosity. Psychonomic Science, 24, 71-72.

Mo, S. S. (1974). Comparative judgment of temporal duration as a function of numerosity. Bulletin of the Psychonomic Society, 3, 377-379.

Mo, S. S. (1975). Temporal reproduction of duration as a function of numerosity. Bulletin of the Psychonomic Society, 5, 165-167.

Moore, C. M., Yantis, S., \& Vaughan, B. (1998). Object-based visual selection: Evidence from perceptual completion. Psychological Science, 9, 104-110.

Nakayama, K., \& Mackeben, M. (1989). Sustained and transient components of focal visual attention. Vision Research, 29, 1631-1649.

Neely, C. A., \& Dagenbach, D. (1996, November). Exogenous and endogenous cueing: Spatial versus object-based visual attention. Poster session presented at the 37th Annual Meeting of the Psychonomic Society, Chicago.

Posner, M. I., Snyder, C. R. R., \& Davidson, B. J. (1980). Attention and the detection of signals. Journal of Experimental Psychology: General, 109, 106-174.

Predebon, J. (1996a). The effects of active and passive processing of interval events on prospective and retrospective time estimates. Acta Psychologica, 94, 41-58.

Predebon, J. (1996b). The relationship between the number of presented stimuli and prospective duration estimates: The effect of concurrent task activity. Psychonomic Bulletin \& Review, 3, 376-379.

Prinzmetal, W., Amiri, H., Allen, K., \& Edwards, T. (1998). Phenomenology of attention: 1. Color, location, orientation, and spatial frequency. Journal of Experimental Psychology: Human Perception \& Performance, 24, 261-282.

Prinzmetal, W., \& Wilson, A. (1997). The effect of attention on phenomenal length. Perception, 26, 193-205.

Shaw, M. L., \& SHaw, P. (1977). Optimal allocation of cognitive resources to spatial locations. Journal of Experimental Psychology: Human Perception \& Performance, 3, 201-211.

Stelmach, L. B., \& Herdman, C. M. (1991). Directed attention and perception of temporal order. Journal of Experimental Psychology: Human Perception \& Performance, 17, 539-550.

Stelmach, L. B., Herdman, C. M., \& McNeil, K. R. (1994). Attentional modulation of visual processes in motion perception. Journal of Experimental Psychology: Human Perception \& Performance, 20, 108-121.

STroop, J. R (1935). Studies of interference in serial verbal reactions. Journal of Experimental Psychology, 18, 624-643.

Thomas, E. A. C., \& Weaver, W. B. (1975). Cognitive processing and time perception. Perception \& Psychophysics, 17, 363-367.

Witherspoon, D., \& Allan, L. G. (1985). The effect of a prior presentation on temporal judgments in a perceptual identification task. Memory \& Cognition, 13, 101-111.

Yeshurun, Y., \& CARrasco, M. (1998). Attention improves or impairs visual performance by enhancing spatial resolution. Nature, 396, 7275.

ZAKAY, D. (1993). Relative and absolute duration judgments under prospective and retrospective paradigms. Perception \& Psychophysics, 54, 656-664.

ZAKAY, D. (1998). Attention allocation policy influences prospective timing. Psychonomic Bulletin \& Review, 5, 114-118.

(Manuscript received October 22, 1999; revision accepted for publication December 21,2000.) 